\title{
Birth of thoracic surgery in Japan
}

\author{
Akira Masaoka, MD, PhD
}

(C) The Japanese Association for Thoracic Surgery 2008

\section{Erratum to: Gen Thorac Cardiovasc Surg (2008) 56:3-9 DOI 10.1007/s11748-007-0150-6}

The name under the photograph in the bottom center of Fig. 3 appeared incorrectly in the article cited above. The correct name should be given as Dr. Chigashi Suzuki, not Dr. Shigashi Suzuki. The publisher sincerely apologizes for the error.

This online version of the original article can be found at http://dx.doi.org/10.1007/s11748-007-0150-6.

A. Masaoka $(\bowtie)$

Emeritus Professor of Surgery, Nagoya City University,

Kawasumi 1, Mizuho-cho, Mizuho-ku, Nagoya 467-8601, Japan Tel. +81-52-853-8231; Fax +81-52-853-6440

e-mail (secretary): tenpaku@med.nagoya-cu.ac.jp 